\title{
Valuation of European and American Option Prices Under the Levy Processes with a Markov Chain Approximation
}

\author{
Gyu-Sik Han* \\ Division of Business Administration, Chonbuk National University
}

(Received: October 24, 2013 / Revised: October 27, 2013 / Accepted: October 28, 2013)

\begin{abstract}
This paper suggests a numerical method for valuation of European and American options under the two Lévy Processes, Normal Inverse Gaussian Model and the Variance Gamma model. The method is based on approximation of underlying asset price using a finite-state, time-homogeneous Markov chain. We examine the effectiveness of the proposed method with simulation results, which are compared with those from the existing numerical method, the latticebased method.
\end{abstract}

Keywords: Markov Chain, Normal Inverse Gaussian model, Variance Gamma Model, European Option, American Option

* Corresponding Author, E-mail: gshan0815@jbnu.ac.kr

\section{INTRODUCTION}

As the computer technology to gather data has developed rapidly, the practitioners or the academicians in finance have been interested in analyzing the financial market data. The Black-Scholes model, which is based on the geometric Brownian motion, succeeded greatly in explaining the financial asset price movement and valuing options, and still is used much recently Black and Scholes (1973). However, the Black-Scholes model cannot reflect some important features often observed in the high-frequency data, the transaction data made every less than 5 minutes. For instance, they are negative skewness, heavy-tailed feature, volatility clustering effect, and discontinuity in the empirical distribution of asset returns. In addition, the Black-Scholes assumption on constant volatility is incompatible with market because a numerical inversion of the Black-Scholes equation based on option market prices from different strikes and fixed maturity, produces a so-called volatility skew or smile. To capture these features, a lot of authors have recently proposed the use of infinite-activity pure jump
Lévy processes for the process describing the dynamics of the asset's log price. Further, it is proposed by Geman et al. (2001) that such processes may be regarded as Brownian motion subordinated to a random clock. At an empirical level, Carr et al. (2003) presents evidence supporting the view that in the presence of an infinite activity Lévy process one do not need a diffusion component. There are three typical examples of infinite activity pure jump Lévy processes. First, we have the normal inverse Gaussian model of Barndorff-Nielsen (1997), and its generalization to the generalized hyperbolic class by Eberlein et al. (1998). Second, we have the variance gamma model studied by Madan and Seneta (1990), Madan and Milne (1991), Madan et al. (1998). Finally, we have the CGMY model, the generalized variance gamma model, which was developed by Carr et al. (2003).

In infinite activity Lévy processes, numerical derivative valuation is approached in various ways. Partial-integro differential equations (PIDEs) are widely used Cont and Voltchkova (2005), Hirsa and Madan (2004), Matache et al. (2005). It is another approach to compute directly the risk-neutral expectation of the pay- 
off at expiry. For instance, inversion of Fourier transform or Monte Carlo methods can be used Carr and Madan (1999), Fu et al. (2001), Glasserman, (2003). A third alternative utilizes lattice-based methods. Cox et al. (1979) firstly proposed a binomial tree model for approximation of the continuous time Black-Scholes model and the backward option pricing. This approach was extended to the finite activity setting of the jump diffusion models. (Amin, 1993; Mulinacci, 1996); Finite activity refers to the underlying Lévy process having only many (but finite) discontinuities per unit time. More generally, Këllezi and Webber (2004) and Maller et al. (2006) suggested a lattice method for an infinite activity Lévy case. And Han (2012) and Simonato (2011) proposed the option pricing method based on a Markov chain under a Lévy process very lately. Simonato (2011) applied the method only to the Merton's jump diffusion model because the transition density, the density of the logarithmic stock price change, derives easily in closed form Merton (1976). In Han (2012), since there is no closed form of the transition density in the Kou's double exponential jump diffusion model, the second order approximation of the transition density was calculated and used to value American option prices Kou (2002). So far, there is no study about numerical option pricing with direct use of the stock price distribution in the Lévy processes with infinite activity pure jumps. Therefore, this paper suggests how the Markov chain approach can be used to numerically value option prices. Particularly, we focus on the two main infinite activity models, NIG and VG.

This paper provides the brief summary of the two model and how we can calculate option prices with Markov chain-based method in Section 2 and 3, and numerical experimental results compared with results from Maller et al. (2006) in Section 4. Finally, the conclusion is given in Section 5.

\section{MODEL OVERVIEW}

Let $B(t ; \theta, \sigma)$ be a Brownian with constant drift rate $\theta$ and volatility $\sigma$. That is,

$$
B(t ; \theta, \sigma)=\theta d t+\sigma W(t), W(t) \sim N(0, t)
$$

where $t$ is the deterministic time scale. However, if $t$ is observed on a stochastic time scale, which is called a subordinator, we can define a new process. When a subordinator is an independent gamma process, the new process $(X)$ is the variance gamma (VG) model. Then,

$$
\begin{aligned}
X_{V G}(t ; \theta, \sigma, v) & \equiv B(\gamma(t ; 1, v) ; \theta, \sigma) \\
& =\theta \gamma(t ; 1, v)+\sigma W(\gamma(t ; 1, v))
\end{aligned}
$$

where $\gamma(t ; \mu, v)$ is a gamma process with mean rate $\mu$ and variance rate $v$. The gamma process is defined as the process of independent gamma increments over nonoverlapping intervals of time $(t, t+h)$. The density of the increments $g=\gamma(t+h ; \mu, v)-\gamma(t ; \mu, v)$ is given by the gamma density function with mean rate $\mu h$ and variance $v h$. (See the details in Madan et al. (1998)) Furthermore, the dynamics of the VG model is given by:

$$
\begin{aligned}
f_{t}^{V G}(x ; \theta, \sigma, v)= & \frac{2 \exp \left(\theta x / \sigma^{2}\right)}{v^{t / v} \sqrt{2 \pi} \Gamma(t / v)}\left(\frac{x^{2}}{2 \sigma^{2} / v+\theta^{2}}\right)^{\frac{t}{2 v}-\frac{1}{4}} \\
& \times K_{\frac{t}{v}-\frac{1}{2}}\left(\frac{1}{\sigma^{2}} \sqrt{x^{2}\left(\frac{2 \sigma^{2}}{v}+\theta^{2}\right)}\right)
\end{aligned}
$$

We can obtain the characteristic function of the VG model from Equation (3) and it is as follows:

$$
E\left[e^{i z X_{V G}(t)}\right]=\left(1-i z \theta v+\frac{\theta^{2} v z^{2}}{2}\right)^{-\frac{t}{v}}
$$

In case that a subordinator is an independent inverse Gaussian process, the process is called the normal inverse Gaussian (NIG) model. That is,

$$
\begin{array}{r}
X_{N I G}(t ; \theta, \sigma, v) \equiv B\left(\tau_{t}^{v} ; \theta, \sigma\right) \\
=\theta \tau_{t}^{v}+\sigma W\left(\tau_{t}^{v}\right)
\end{array}
$$

where $\tau_{t}^{v}$ is the first time that a Brownian motion with drift $v$ reaches the positive level $t$ (Carr et al., 2003). Then, the dynamics of the NIG model follows:

$$
f_{t}^{N I G}(x ; \alpha, \beta, \delta)=\frac{\alpha}{\pi} \frac{K_{1}\left(\alpha \delta t q\left(\frac{x}{\delta t}\right)\right)}{q\left(\frac{x}{\delta t}\right)} \exp (\delta t \eta+\beta x)
$$

where $q(y)=\sqrt{1+y^{2}}, \delta=\sigma, \eta=\sqrt{\alpha^{2}-\beta^{2}}, \beta=\theta / \sigma^{2}, \alpha^{2}$ $/ \sigma^{2}+\theta^{2} / \sigma^{4}$. We can also calculate the characteristic function of the NIG model:

$$
E\left[e^{i z X_{N G}(t)}\right]=\exp \left\{t \delta\left(\eta-\sqrt{\alpha^{2}-(\beta+i z)^{2}}\right)\right\}
$$

In both cases, $K_{n}(z)$ means the modified Bessel function of the second kind with order of $n$.

\section{PROPOSED METHOD: OPTION VALUATION WITH A MARKOV CHAIN}

\subsection{Construction of a Markov}

As already known, the risk-neutral process for the underlying asset price is expressed under Lévy processes of our interest as follows: 


$$
S_{t}=S_{0} \exp [(r-q+w) t+X \cdot(t ; \theta, \sigma, v)], t>0
$$

where $w$ is a compensator term. Let us define

$$
\phi_{t}(z)=e^{i z w t} E\left[e^{i z X \cdot(t)}\right]
$$

The $w$ is determined by $\phi_{t}(-i)=1$. Changing Equation (8) with logarithmic prices, we obtain

$$
\ln \left(S_{t} / S_{0}\right)-(r-q+w) t=X \cdot(t ; \theta, \sigma, v)
$$

Thus, we can use Equation (3) or (6) directly.

Before option valuation in the context of the two aforementioned Lévy processes, we will propose how to construct a homogeneous Markov chain in discrete time. Duan and Simonato (2001) has already argued that this chain can converge to the true density of a stochastic process as the number of states increases over the discrete time points. In order to make a Markov chain under the Lévy processes of our interest, let us at first define a column vector whose elements are $n$ logarithmic stock prices possible in the future:

$$
\Upsilon=\left[\begin{array}{llll}
s_{1} & s_{2} & \cdots & s_{n}
\end{array}\right]^{T}, s_{j}=\ln S_{0}+\frac{2 j-n-1}{n-1} I_{\Upsilon}
$$

In Equation (9), $\ln S_{0}$ is the current logarithmic underlying asset price and, since the positive integer $j$ ranges from 1 to $n, s_{1}$ is equal to $\ln S_{0}-I_{\Upsilon}$ and $s_{n}$ is $\ln S_{0}+I_{\Upsilon}$. Moreover, $n$ needs to be only an odd number because $s_{(n+1) / 2}$ becomes the current logarithmic price $\left(\ln S_{0}\right)$ and it is possible to compute an option price at the current asset price level. The quantity $I_{\Upsilon}$ is used to produce a fluctuation of the logarithmic price and calculated using the conditional standard deviation of the price's log return with the option's time to expiry $(\tau)$ in the following way:

$$
I_{\Upsilon}=p(n) \times v \times \sqrt{\tau}
$$

In Equation (10), v, the annualized standard deviation, is possible to compute in the VG model and NIG model:

$$
v_{V G}^{2}=\theta^{2} v+\sigma^{2}, \quad v_{N I G}^{2}=\frac{\delta \alpha^{2}}{\eta^{3}}
$$

$p(n)$, a scaling factor, is an increasing function of $n$, but should satisfy some mild partition conditions that $p(n)<$ $n$ for all positive integer $n$ (Duan and Simonato, 2001).

The transition probability matrix $(M)$ describes the transition probabilities from one state (an underlying asset price) to the other over a discrete unit time. To build the matrix, we define $n$ logarithmic asset price cells following:

$$
\begin{gathered}
C_{j}=\left[c_{j}, c_{j+1}\right) \\
\text { s.t. } c_{1}=-\infty, c_{n+1}=+\infty, c_{j}=\frac{s_{j}+s_{j-1}}{2}(2 \leq j \leq n)
\end{gathered}
$$

Using Equation (9) and (11), the transition probabilities of leaving the current price $\left(s_{i}\right)$ and reaching the price cell $\left(C_{j}\right)$ after a discrete unit time are defined as $m_{i, j}(\in M)$. They are computed with integration of Equation (3) and (6):

$$
\begin{aligned}
& m_{i, j}=\operatorname{Pr}\left(C_{j} \mid s_{i}\right)=F^{A}\left(c_{j}-s_{i}, c_{j+1}-s_{i}\right) \\
& F^{A}\left(r_{1}, r_{2}\right)=\int_{r_{1}}^{r_{2}} f^{A}(x) d x\left(r_{1}<r_{2}\right)
\end{aligned}
$$

where $f^{A}(x)$ is the probability density function of a stochastic process $A$. Therefore, $M$ is:

$$
M=\left[\begin{array}{ccccc}
m_{1,1} & m_{1,2} & \cdots & m_{1, n-1} & m_{1, n} \\
m_{2,1} & m_{2,2} & \cdots & m_{2, n-1} & m_{2, n} \\
\vdots & \vdots & \ddots & \vdots & \vdots \\
m_{n-1,1} & m_{n-1,2} & \cdots & m_{n-1, n-1} & m_{n-1, n} \\
m_{n, 1} & m_{n, 2} & \cdots & m_{n, n-1} & m_{n, n}
\end{array}\right]
$$

As mentioned previously, Equations from (9) to (13) show that, as $n$ goes to infinity, the change of logarithmic asset prices can be expressed better and $M$ is more similar to the target stochastic process of our interest. Equation (13) appears for us to totally have $n^{2}$ computations, but we do not actually. Because, as seen in Equation (9), all the differences between two consecutive elements in the column vector are the same, the transition probability $m_{i, j}$ is not always different for each $i$ and $j$. That is, some elements in $M$ are the same with one another and some elements can be induced from other elements already known. For example, $M$ with $n=7$ follows:

$$
M=\left[\begin{array}{ccccccc}
m_{1,1} & m_{1,2} & m_{1,3} & m_{1,4} & m_{1,5} & m_{1,6} & m_{1,7} \\
\Lambda_{2,1} & m_{2,2} & m_{1,2} & m_{1,3} & m_{1,4} & m_{1,5} & \Psi_{2,7} \\
\Lambda_{3,1} & m_{7,6} & m_{2,2} & m_{1,2} & m_{1,3} & m_{1,4} & \Psi_{3,7} \\
\Lambda_{4,1} & m_{7,5} & m_{7,6} & m_{2,2} & m_{1,2} & m_{1,3} & \Psi_{4,7} \\
\Lambda_{5,1} & m_{7,4} & m_{7,5} & m_{7,6} & m_{2,2} & m_{1,2} & \Psi_{5,7} \\
\Lambda_{6,1} & m_{7,3} & m_{7,4} & m_{7,5} & m_{7,6} & m_{2,2} & \Psi_{6,7} \\
m_{7,1} & m_{7,2} & m_{7,3} & m_{7,4} & m_{7,5} & m_{7,6} & m_{7,7}
\end{array}\right]
$$

where $\Lambda_{k, 1}=\sum_{j=1}^{8-k} m_{7, j}$ and $\Psi_{k, 7}=\sum_{j=8-k}^{7} m_{1, j}$. As seen in the above transition probability matrix, all the elements on the diagonal line are equal except for two elements, the first and last elements $\left(m_{1,1} \& m_{7,7}\right)$, all the elements above the diagonal line can be computed with the elements from the first row of the matrix, and all the elements below the diagonal line can be computed from the elements of the last row. Thus, the total number of computations in the matrix is not $n^{2}$ but $2 n+1$ and the computational complexity is efficiently linear. It should also be noticed that the probabilities of the first line are monotonically decreasing while those from the last line are monotonically increasing. 


\subsection{Option Valuation with a Markov Chain}

In this section, we propose how to price an option with a constructed Markov Chain. Similarly to the conventional lattice method or FDM with PIDEs, we utilize the recursive valuation beginning from an option maturity. Let us consider an option with $T$ years to maturity and exercise price $K$, and define a discrete unit time $h(=$ $\Delta t$ ) to be $T / L$ as follows:

$$
t_{0}, t_{1}, t_{2}, \cdots, t_{L-1}, t_{L} \Rightarrow \Delta t=t_{i}-t_{i-1}, t_{0}=0, t_{L}=T
$$

Then, at maturity $T$,

$$
O V(\Upsilon, T)=\max \left[\omega\left(e^{\Upsilon}-K \cdot \mathbf{1}_{\mathbf{v}}\right), \mathbf{0}_{\mathbf{v}}\right]
$$

$\Upsilon$ follows the definition of Equation (9), and $\mathbf{1}_{\mathrm{v}}$ and $\mathbf{0}_{\mathrm{v}}$ are the $n$-dimensional column vectors of ones and zeros, respectively. $\omega$ denotes a vanilla call $(\omega=1)$ or a vanilla put $(\omega=-1)$. Moreover, $\max [\cdot, \cdot]$ is defined as a function of element-wise operation. Hence, $O V(\Upsilon, T)$ becomes a $n$-dimensional column vector. At time $\tau$ before maturity ( $\tau=j \cdot h, j=0,1, \cdots, L-1$ ), we need to think what style an option is because its valuation depends on the style. In this paper, we deal with two styles, European and American. If the option is European, it is never exercised until maturity, and therefore $O V(\Upsilon, \tau)$ is:

$$
O V(\Upsilon, \tau)=e^{-r h} \times M \times O V(\Upsilon, \tau+h)
$$

where $r$ is a risk-free rate. If it is American, since it can be exercised at time $\tau$,

$$
\begin{array}{r}
O V(\Upsilon, \tau)=\max \left[\max \left\{\omega\left(e^{\Upsilon}-K \cdot \mathbf{1}_{\mathbf{v}}\right), \mathbf{0}_{\mathbf{v}}\right\},\right. \\
\left.e^{-r h} \times M \times O V(\Upsilon, \tau+h)\right]
\end{array}
$$

$\max \left\{\omega\left(e^{\Upsilon}-K \cdot \mathbf{1}_{\mathbf{v}}\right), \mathbf{0}_{\mathbf{v}}\right\}$ is the instantaneous exercise value at time $\tau$ and $e^{-r h} \times M \times O V(\Upsilon, \tau+h)$ is the value to hold the option until time $\tau+h$ without its exercise at time $\tau$. Continuing to compute (17) or (18) recursively until time 0 , we obtain $O V(\Upsilon, 0)$, which is also a $n$ dimensional column vector. The $(n+1) / 2$-th element of $O V(\Upsilon, 0)$ is the option value at the current asset price level. It should be noticed in Equation (17) and (18) that we expect to obtain an option value closer to the true value as $n$ increases.

\section{EXPERIMENTAL RESULTS}

Depending on the proposed method, we experiment on valuation of a European vanilla put and an American vanilla put, and compare our results with those in Carr and Madan (1999) and Maller et al. (2006). The experimental common conditions are:

$$
\begin{aligned}
& S_{0}=100, r=0.1, q=0 \\
& T=\{0.25,1.00\}, K=\{90,95,100,105,110\}
\end{aligned}
$$

Both $r$ and $q$ are on annual basis. The parameters of the VG model and the NIG model in our experiments are as follows:

$$
\begin{aligned}
& \text { VG: } \theta=-0.14, \sigma=0.12, v=0.2 \\
& \text { NIG: } \alpha=28.421, \beta=-15.086, \delta=0.317
\end{aligned}
$$

The above parameters are introduced in Madan (1999) and Maller et al. (2006). All of them are also on annual basis. Moreover, $p(n)$ is set to be $\ln (n)$ and, if $m_{i, j}$ is equal to or less than our threshold $\left(10^{-10}\right)$, it will be zero. The discrete unit time $(h)$ is set to be $h=1 / 364$.

European vanilla put values using our proposed method, Madan (1999), and Maller et al. (2006) are denoted by E-MC, E-FFT, and E-MN, respectively. Similarly, American vanilla put values depending on our proposed method and Maller et al. (2006) are A-MC and A$\mathrm{MN}$, respectively. The ER(error rate) between our proposed method and benchmark methods in Madan (1999) and Maller et al. (2006) is on per cent and defined, in the European style, as:

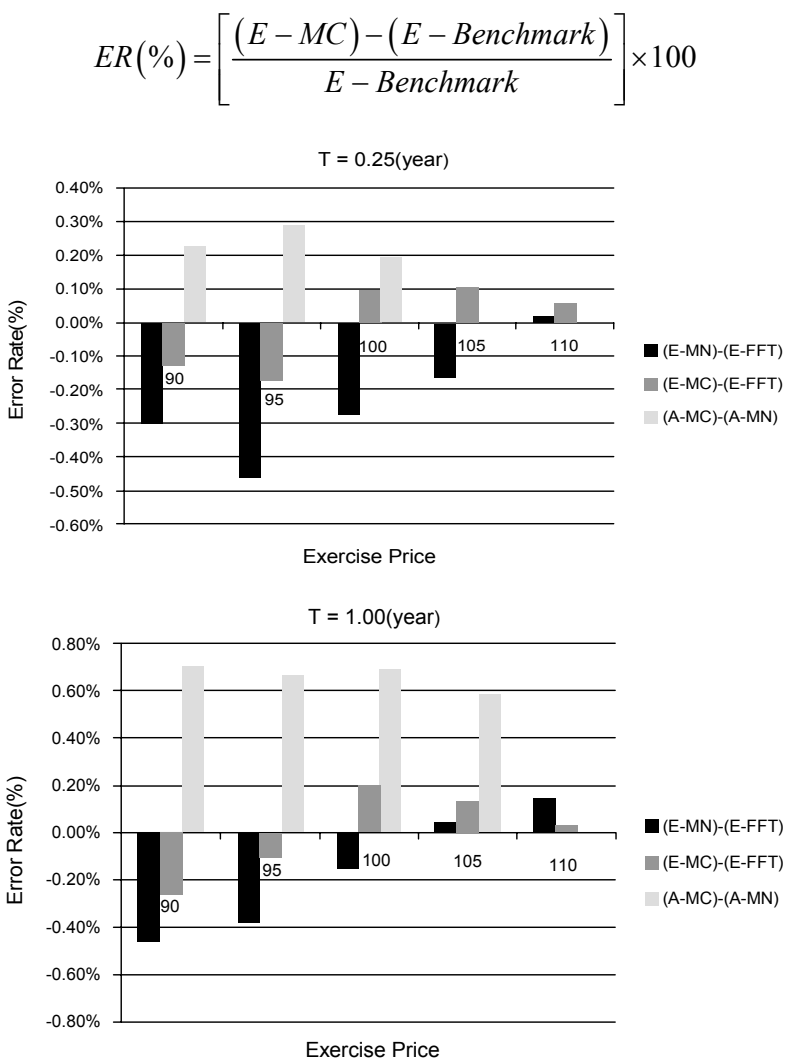

Figure 1. Comparison of ERs in the VG Model

The results about the VG model are shown in Figure 1 and those about the NIG model are in Figure 2. In case of the European vanilla puts, E-FFT is the benchmark 
value and we calculate the ERs of E-MC and E-MN, respectively. As shown in Figure 1 and 2, ERs of both E$\mathrm{MC}$ and E-MN are all negative, but the ERs of E-MC are less than those of E-MN in absolute magnitude. That is, our proposed method shows more accuracy in pricing them. Moreover, the lattice method proposed by Maller et al. (2006) tends to undervalue the European vanilla puts compared with our proposed method. This trend appears to happen in the American vanilla puts because the ERs between A-MC and A-MN are all positive. We omit the detailed results about Figure 1 and 2 .

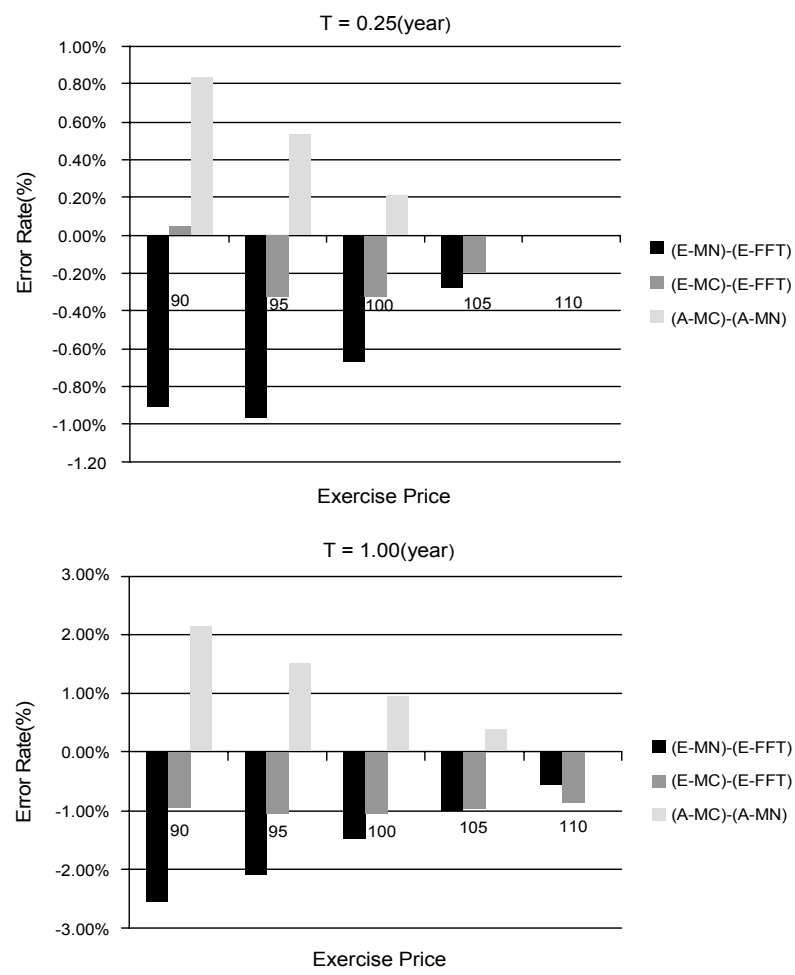

Figure 2. Comparison of ERs in the NIG Model

\section{CONCLUSIONS}

In this paper, we study how to value options with an approximate Markov chain under two infinite activity Lévy processes, VG model and NIG model. Our proposed method is based on the known density of the models and yields a simple approach easy to understand compared with various approaches (lattice methods, FDM, inversion of Fourier transform) in the literature. From the experimental results, when we increase by the enough number of states (asset prices), our proposed method shows that it produces option values closer to the true values in both European and American styles. We need a further research beyond this study. It is whether our proposed method can be applied to the Lévy processes that have no density in closed form. We will propose how to overcome the drawback in the future research.

\section{ACKNOWLEDGEMENT}

This research was supported by the Basic Science Research Program through the National Research Foundation of Korea (NRF) funded by the Ministry of Science, ICT, and Future Planning (No. 2013R1A1A1010404).

\section{REFERENCES}

Amin, K., "Jump-diffusion Option Valuation in Discrete Time,” Journal of Finance 48 (1993), 1833-1863.

Barndorff-Nielsen, O. E., "Normal Inverse Gaussian Distribution and Stochastic Volatility Modelling," Scandinavian Journal of Statistics 24 (1997), 1-13.

Black, F. and M. Scholes, "The Pricing of Options and Corporate Liabilities," Journal of Political Economy 81 (1973), 637-659.

Carr, P., H. Geman, D. B. Madan, and M. Yor, "Stochastic Volatility for Lévy Processes," Mathematical Finance 13 (2003), 345-382.

Carr, P. and D. B. Madan, "Option Valuation using the fast Fourier Transform," Journal of Computational Finance 2 (1999), 61-73.

Cont, R. and E. Voltchkova, "Integro-Differential Equations for Option Prices in Exponential Lévy Models," Finance Stochastics 9 (2005), 299-325.

Cox, J. C., S. A. Ross, and M. Rubinstein, "Option Pricing: A Simplified Approach," Journal of Financial Economics 3 (1979), 125-144.

Duan, J. C. and J.-G. Simonato, “American Option Pricing under GARCH by a Markov Chain Approximation," Journal of Economic Dynamics and Control 25 (2001), 1689-1718.

Eberlein, E., U. Keller, and K. Prause, "New Insights into Smile, Mispricing and Value at Risk," Journal of Business 71 (1998), 371-406.

Fu, M. C., S. B. Laprise, D. B. Madan, Y. SU, and R. WU, "Pricing American Options: A Comparison of Monte Carlo Simulation Approaches," Journal of Computational Finance 2 (2001), 62-73.

Geman, H., D. B. Madan, and M. Yor, "Time Changes for Lévy Processes," Mathematical Finance 11 (2001), 79-96.

Glasserman, P., Monte Carlo Methods in Financial Engineering, Springer, New York, 2003.

Han, G. S., "Valuation of American Option Prices Under the Double Exponential Jump Diffusion Model with a Markov Chain Approximation," Journal of the Korean Institute of Industrial Engineer 38 (2012), 249-253.

Hirsa, A. and D. B. Madan, "Pricing American Options under Variance Gamma," Journal of Computational Finance 7 (2004), 63-80. 
Këllezi, E. and N. Webber, "Valuing Bermudan Options When Asset Returns Are Lévy Processes," Quantitative Finance 4 (2004), 87-100.

Kou, S. G., "A Jump-diffusion Model for Option Pricing," Management Science 48 (2002), 1086-1101.

Madan, D. B. and E. Seneta, "The Variance Gamma (VG) Model for Share Market Returns," Journal of Business 63 (1990), 511-524.

Madan, D. B. and F. Milne, "Option Pricing with VG Martingale Components," Mathematical Finance 1 (1991), 39-56.

Madan, D. B., P. Carr, and E. Chang, "The Variance Gamma Process and Option Pricing," European Finance Review 2 (1998), 79-105.

Maller, R., D. H. Solomon, and A. Szimayer, "A Multinomial Approximation for American Option Prices in Lévy Process Models," Mathematical Finance 16 (2006), 613-633.

Matache, A.-M., P. A. Nitsche, and C. Schwab, "Wavelet Galerkin Pricing of American Options on Lévy Driven Assets," Quantitative Finance 5 (2005), 403424.

Merton, R. C., "Option Pricing When the Underlying Process for Stock Returns is Discontinuous," Journal of Financial Economics 3 (1976), 124-144.

Mulinacci, S., "An Approximation of American Option Prices in a Jump-Diffusion Model," Stochastic Processes Application 62 (1996), 1-17.

Simonato, J.-G., "Computing American Option Prices in the Lognormal Jump-diffusion Framework with a Markov Chain," Finance Research Letters 8 (2011), 220-226. 